\author{
Z. Bengaly ${ }^{1}$ \\ P.H. Clausen ${ }^{2}$ \\ H. Boly ${ }^{3}$ \\ A. Kanwe ${ }^{1}$ \\ G. Duvallet ${ }^{1}$
}

\section{Comparaison de la trypanosomose expérimentale chez certaines races de petits ruminants au Burkina Faso}

BENGALY (Z.), CLAUSEN (P.H.), BOLY (H.), KANWE (A.), DUVALLET (G.). Comparaison de la trypanosomose expérimentale chez certaines races de petits ruminants au Burkina Faso. Revue Élev. Méd. vét. Pays trop.., 1993, 46 (4) : 563-570

Pour comparer leurs degrés respectifs de sensibilité à l'égard de la trypanosomose, 24 moutons et chèvres de race naine Djallonké, et 16 moutons et chèvres Peul du Sahel, ont été inoculés avec des souches de Trypanosoma vivax et de Trypanosoma congolense. Un animal de chaque race a servi de témoin. Une anémie est observée chez tous les animaux inoculés. Pour l'infection à $T$. vivax , 1 chèvre Djallonké sur 6,3 chèvres sur 4 et 2 moutons sur 4 de race Peul du Sahel sont morts en 16 semaines d'expérimentation. Une seule mortalité sur 4 est enregistrée dans le lot des moutons Peul du Sahel, pour l'infection à $T$. congolense. Les chutes de poids et de concentration leucocytaire observées ne diffèrent pas significativement entre Djallonké et Peul du Sahel durant 8 semaines. Cette étude montre une bonne résistance des moutons et chèvres Djallonké à l'infection à $T$. vivax. Par contre, il n'y a pas de différence significative entre les deux races de moutons et de chèvres infectés à $T$. congolense.

Mots-clés : Caprin - Ovin - Chèvre Djallonké - Chèvre Peul - Mouton Djallonké - Mouton Peul - Trypanosomose - Trypanosoma congolense Trypanosoma vivax - Trypanotolérance - Infection expérimentale - Burkina Faso.

\section{INTRODUCTION}

La trypanosomose animale est une contrainte à la promotion de l'élevage des petits ruminants en Afrique au sud du Sahara. Au Burkina Faso, une enquête de DISSET (5) le confirme.

Cependant, on remarque que les moutons et chèvres de race naine Djallonké vivent bien dans les zones subhumides infestées de glossines sans qu'apparemment la trypanosomose soit une contrainte majeure. En revanche, les moutons et chèvres de race Peul du Sahel meurent à plus ou moins brève échéance lorsqu'ils sont acheminés dans ces régions. Ces observations ont conduit à les considérer comme trypanosensibles comparativement aux premiers qualifiés de trypanotolérants ; mais on dispose de peu de renseignements précis sur leurs degrés respectifs de sensibilité.

1.Centre de recherches sur les trypanosomoses animales (CRTA), 01 B.P. 454 , Bobo-Dioulasso 01, Ouagadougou 03, Burkina Faso.

2. Service Labor IN, BGA, FII 4 Postfach 330013, D-1000 Berlin 33, Allemagne.

3. Institut du développement rural (IDR), Université de Ouagadougou, B.P. 7021, Burkina Faso.

Reçu le 20.10.1992, accepté le 11.5.1993.
C'est dans ce sens que le Centre de recherches sur les trypanosomoses animales (CRTA) a entrepris des études de pathologie comparée entre les races Djallonké du sud du Burkina Faso (Pays Lobi) et celles du nord (Sahel) pour contribuer à la connaissance de l'épidémiologie de cette maladie.

\section{MATÉRIEL ET MÉTHODES}

\section{Troupeau expérimental}

Le troupeau est constitué de 44 animaux âgés de 1 à 2 ans. Huit groupes ont été constitués : quatre de 6 Djallonké chacun, et quatre de 4 Peul du Sahel chacun. Chaque groupe est composé d'animaux de même espèce et race. Un animal de chaque race a servi de témoin. Les animaux de race Djallonké ont été achetés chez des éleveurs au "Pays Lobi", zone infestée par les glossines (3); ceux du Sahel proviennent de Djibo, au nord du Burkina Faso, en dehors de la zone de répartition des glossines.

\section{Infection}

Les animaux ont été traités à leur arrivée au Centre avec un trypanocide (acéturate de diminazène* ; $7 \mathrm{mg} / \mathrm{kg}$ ), un antibiotique (oxytétracycline ${ }^{\star \star}$ ) et un déparasitant interne (fenbendazole ${ }^{\star \star \star}$ ). Une mise en quarantaine de 6 mois pour les Djallonké et de 2 mois pour les Peul a été observée. Les 44 animaux retenus sur l'effectif initial de 56 ont donné une réponse négative au test de détection des antigènes circulants de trypanosomes.

L'infection a été effectuée en injectant par voie intraveineuse $10^{6}$ trypanosomes par animal. Les souches de $T$. congolense et de T. vivax utilisées appartiennent respectivement aux stocks Karankasso/83/CRTA/57 et Satiri/87/CRTA/134.

\footnotetext{
${ }^{*}$ Berenil ${ }^{\otimes}$

** Terramycin/LA ${ }^{\oplus}$

${ }^{\star \star *}$ PANACUR Ovin ${ }^{\circledast}$
} 


\section{Z. Bengaly P.H. Clausen H. Boly A. Kawne G. Duvallet}

\section{Suivi des animaux}

Ce suivi a consisté à observer régulièrement les signes cliniques, les variations de poids, de l'hématocrite, des concentrations leucocytaires, la parasitémie et la réponse immunitaire par test ELISA. Le canevas expérimental est résumé dans le tableau I. Certains paramètres n'ont été suivis que pendant 2 mois seulement pour des raisons logistiques.

La parasitémie est estimée en examinant l'interface cellules-plasma entre lame et lamelle en contraste de phase sur 40 champs, après centrifugation différentielle en tubes capillaires (6). La numération leucocytaire est effectuée à l'aide de la cellule hématimètre de Neubauer améliorée, après dilution du sang au 1/20 dans l'acide acétique 3 p. 100 (4). Les anticorps IgM et IgG sont dosés selon la technique ELISA améliorée par BOCQUENTIN et DUVALLET (1).

L'antigène est préparé au CRTA avec une souche de $T$. evansi isolée sur dromadaire au Kenya. La différence observée dans ce test entre antigènes homologues et hétérologues est minime : ce sont les antigènes communs qui sont en jeu. Aussi le choix de T. evansi résultet-il de la grande virulence de cette souche pour les souris et de la possibilité d'obtention rapide d'une grande quantité d'antigènes.

\section{TABLEAU I Canevas expérimental.}

\begin{tabular}{|l|c|c|}
\hline & Rythme du suivi & $\begin{array}{c}\text { Durée } \\
\text { (mois) }\end{array}$ \\
Examen clinique $\left(\mathrm{T}^{\circ} \mathrm{C}\right)$ & quotidien & 4 \\
Hématocrite & quotidien & 2 \\
Poids & hebdomadaire & 2 \\
Parasitémie & quotidien & 2 \\
Numération leucocytaire & bihebdomadaire & 2 \\
Récolte de sérum & bihebdomadaire \\
Autopsie & en cas de mortalité & 4 \\
\hline
\end{tabular}

\section{Analyse statistique}

La signification statistique, entre lots, des périodes prépatentes, des variations de poids, de l'hématocrite, des parasitémies, des concentrations leucocytaires est évaluée en utilisant le test de Student. Les comparaisons sont effectuées entre races Djallonké et Peul du Sahel de même espèce ayant reçu la même souche de trypanosomes.

\section{RÉSULTATS}

\section{Observations d'ordre clinique}

Les principaux signes cliniques observés sont une fièvre intermittente, de l'inappétence, une démarche chancelante et de l'amaigrissement.

Dans les lots infectés par $T$. vivax, les périodes prépatentes varient entre 5 et 10 jours chez les Djallonké et entre 5 et 7 jours chez les Peul. Trois chèvres et deux moutons de race Peul et une chèvre Djallonké sont morts au cours de l'étude.

Pour l'infection à $T$. congolense, les périodes prépatentes varient entre 4 et 10 jours chez les Djallonké et chez les Peul. Une mortalité est enregistrée dans le lot de moutons Peul. Les investigations autopsiques ont révélé la présence de pétéchies sur le myocarde. II n'y a pas de différence significative entre lots pour les deux types d'infection.

Les animaux Peul présentent une baisse régulière de poids du début jusqu'à la fin du suivi. Les animaux Djallonké perdent également du poids durant les cinq premières semaines, puis l'on observe une stabilisation (fig.1). A la fin du suivi, les différences observées entre les moyennes des pertes de poids sont non significatives.

\section{Hématocrites}

L'évolution des hématocrites (figure 2) se décompose en une phase de déclin suivie d'une stabilité relative.

Chez les moutons infectés par $T$. vivax, les valeurs des hématocrites chutent de 33 à 24,5 p.100 chez les Djallonké et de 33 à 16 p.100 chez les Peul entre le jour de l'infection et le $20 \mathrm{e}$ jour post-infection. Puis elles varient entre 25 et 28 p. 100 pour les premiers et entre 15 et 23 p.100 pour les seconds. Chez les chèvres infectées par T. vivax, on observe des chutes de 30 à 18 p.100 vers le 32 e jour chez les Djallonké, et de 35 à 16 p.100 vers le $28 \mathrm{e}$ jour chez les Peul. Les hématocrites oscillent ensuite entre 15 et 23 p. 100 .

Dans les lots infectés par $T$. congolense, les hématocrites chutent de 30 à 18 p. 100 chez les moutons Djallonké, et de 40 à 22 p. 100 chez les moutons Peul. A partir du 28e jour post-infection les hématocrites varient entre 20 et 23 p.100. Pour les chèvres, on note des chutes de 33 p. 100 à l'infection à 16 p.100 chez les Djallonké, et de 40 à 16 p. 100 chez les Peul vers le 24 e jour. Puis les hématocrites oscillent entre 15 et 23 p.100.

L'analyse statistique a porté, pour chaque lot, sur les valeurs moyennes des chutes observées entre les valeurs de l'hématocrite à l'infection et les plus basses 
(1)

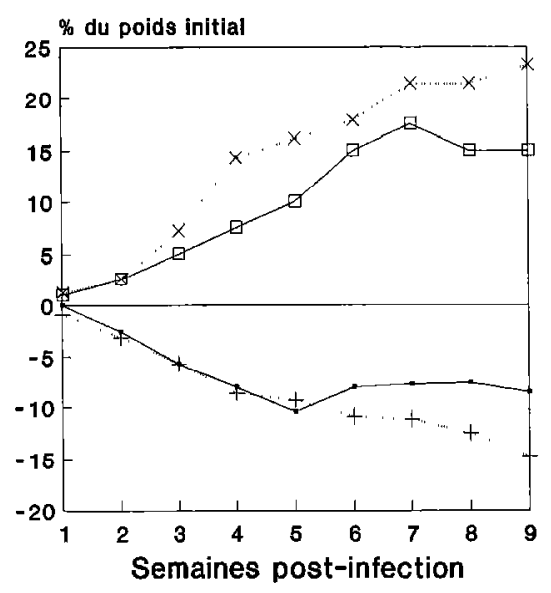

(3)

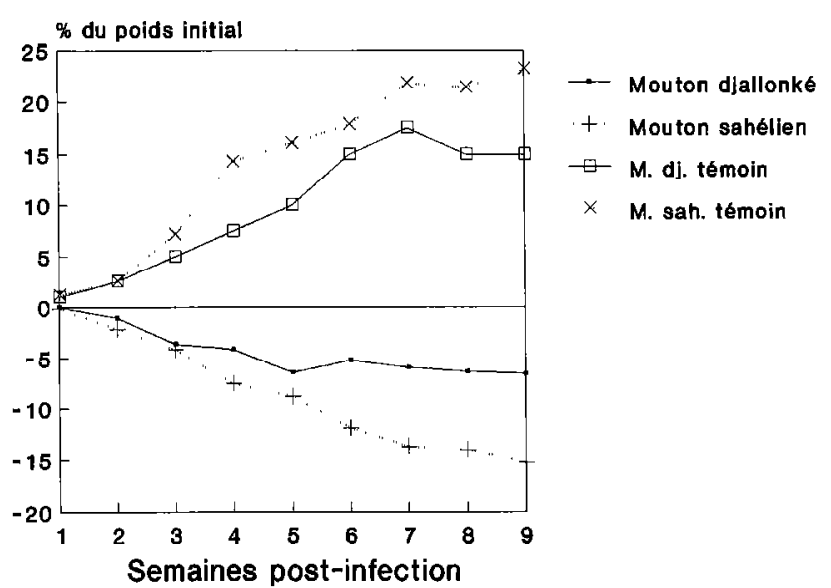

(2)

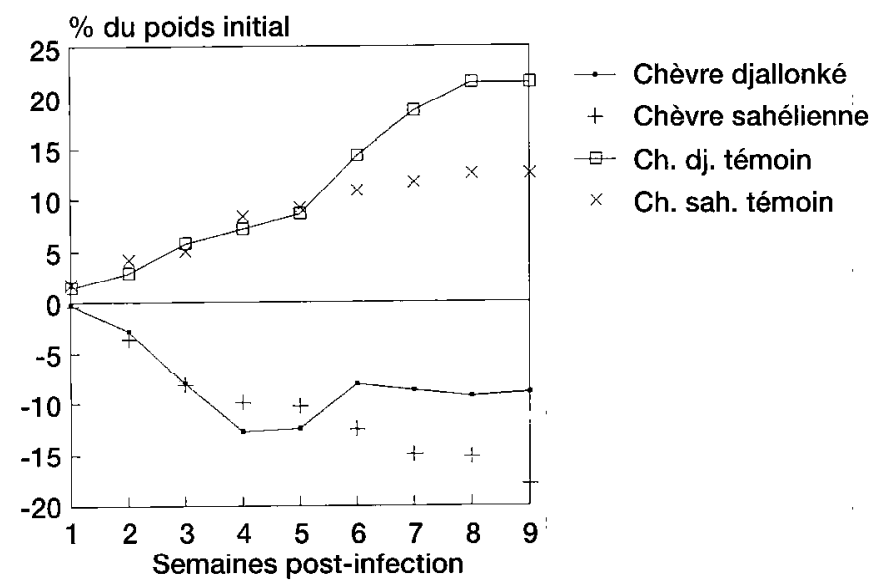

(4)

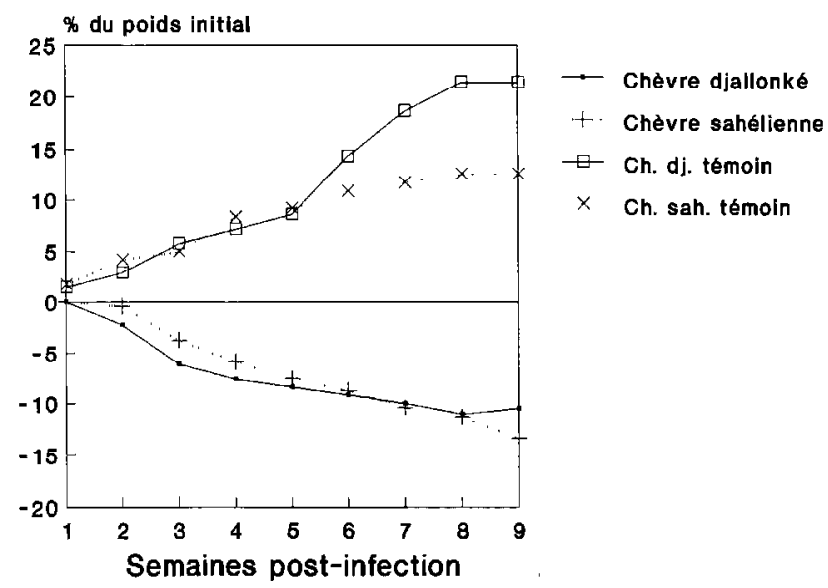

Figure 1 : Variations de poids chez les animaux infectés et les témoins. (1) et (2): infection à $\mathrm{T}$. vivax ; (3) et (4): infection à T. congolense.

mesurées au cours du suivi. L'analyse statistique a aussi porté sur la valeur minimum des moyennes quotidiennes observées (tableau II).

\section{Parasitémies (figure 3)}

Les parasitémies évoluent en dents de scie tout au long du suivi. Elles apparaissent plus faibles chez les Djallonké par rapport aux Peul infectés par $T$. vivax. Le premier pic parasitémique est significativement supérieur $(p<0,05)$ chez les moutons Peul par rapport aux Djallonké. L'analyse statistique a porté sur le nombre de jours de parasitémie détectable exprimé en pourcentage par rapport au nombre de jours de contrôle (tableau III).

\section{Concentration leucocytaire (figure 4)}

Les concentrations leucocytaires diminuent de la première à la cinquième semaine post-infection, puis augmentent et atteignent 150 p.100 des valeurs pré-infection, en particulier chez les moutons Djallonké infectés par $T$. vivax. Les chutes observées atteignent 50 p.100 chez les chèvres Djallonké, les moutons et les chèvres de race Peul infectés par $T$. vivax. II en est de même chez les moutons et chèvres de race Djallonké et chez les chèvres du Sahel infectés par $T$. congolense. La plus forte diminution est de 61,31 p.100 et se rapporte aux chèvres sahéliennes infectées par $T$. vivax. II n'y a pas de différence significative entre Djallonké et Peul pour les deux types d'infection. 
Z. Bengaly P.H. Clausen H. Boly A. Kawne G. Duvallet

(1)

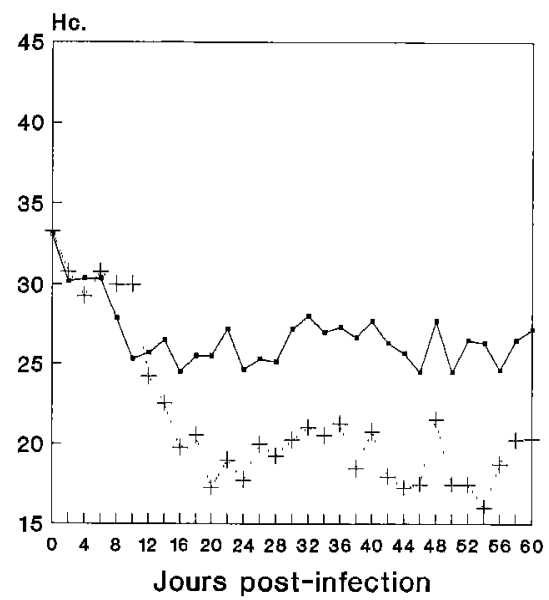

(3)

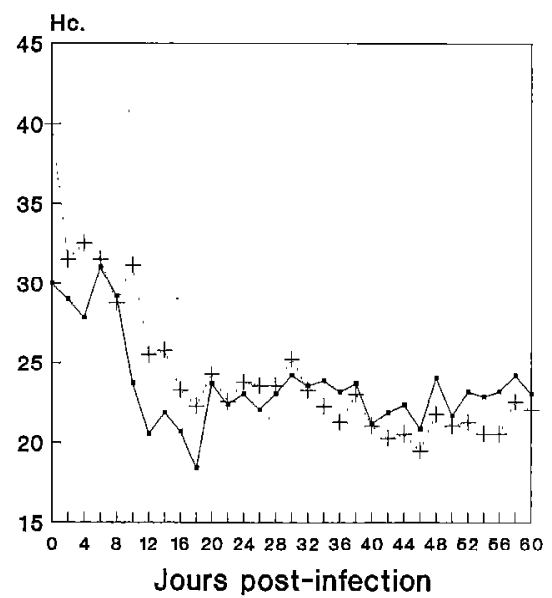

(2)

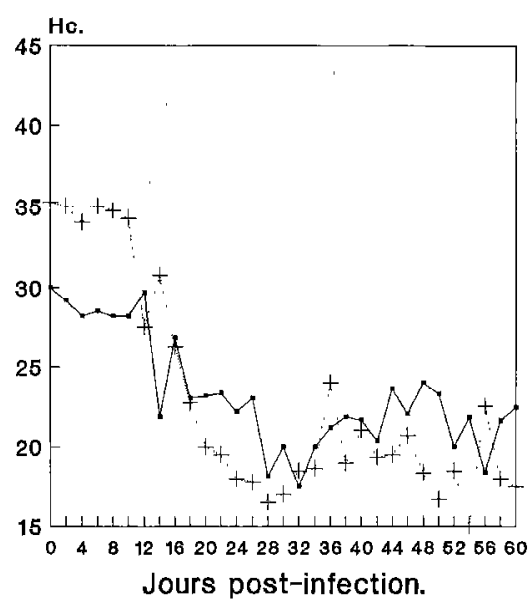

(4)

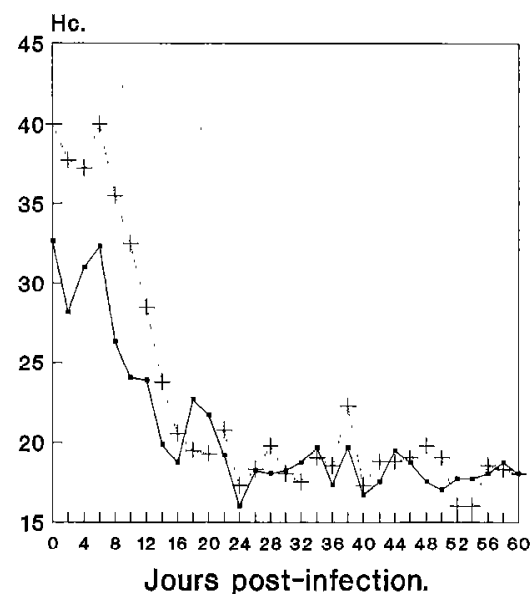

$\rightarrow$ Chèvre djallonké

"+." Chèvre sahélienne
+ Mouton sahélien

- Mouton djallonké

Mouton sahélien
-- Ghèvre djallonké

t. Chère sahélienne

Figure 2 : Evolutions des hématocrites. (1) et (2): infection à T. vivax ; (3) et (4) : infection à T'. congolense.

TABLEAU II Hématocrites : chutes moyennes et valeurs minimums des moyennes quotidiennes pendant la période d'observation.

\begin{tabular}{|c|c|c|c|c|}
\hline Lots & Infection & Chutes moyennes & Minimum & Signification statistique \\
\hline $\begin{array}{l}\text { Mouton Djallonké } \\
\text { Mouton Peul du Sahel }\end{array}$ & $\begin{array}{l}\text { T. vivax } \\
T \text {. vivax }\end{array}$ & $\begin{array}{l}11,66(4,81) \\
21,5(6,10)\end{array}$ & $\begin{array}{l}24,5(9,68) \\
16(6,05)\end{array}$ & $\begin{array}{c}S \\
p<0,05\end{array}$ \\
\hline $\begin{array}{l}\text { Mouton Djallonké } \\
\text { Mouton Peul du Sahel }\end{array}$ & $\begin{array}{l}\text { T. congolense } \\
T \text {. congolense }\end{array}$ & $\begin{array}{l}13(2,71) \\
24(7)\end{array}$ & $\begin{array}{l}20,5(6,24) \\
20,5(4,50)\end{array}$ & NS \\
\hline $\begin{array}{l}\text { Chèvre Djallonké } \\
\text { Chèvre Peul du Sahel }\end{array}$ & $\begin{array}{l}\text { T. vivax } \\
\text { T. vivax }\end{array}$ & $\begin{array}{l}12(4,80) \\
21,5(2,29)\end{array}$ & $\begin{array}{l}18,4(4,59) \\
15(2,82)\end{array}$ & $\begin{array}{c}S \\
p<0,05\end{array}$ \\
\hline $\begin{array}{l}\text { Chèvre Djalonké } \\
\text { Chèvre Peul du Sahel }\end{array}$ & $\begin{array}{l}\text { T. congolense } \\
\text { T. congolense }\end{array}$ & $\begin{array}{l}19,83(3,72) \\
21(5,20)\end{array}$ & $\begin{array}{l}16(6,62) \\
16(1,4)\end{array}$ & NS \\
\hline
\end{tabular}

()$=$ écart-type.

$S:$ significatlf; NS : non slgniflcatif. 
(1)

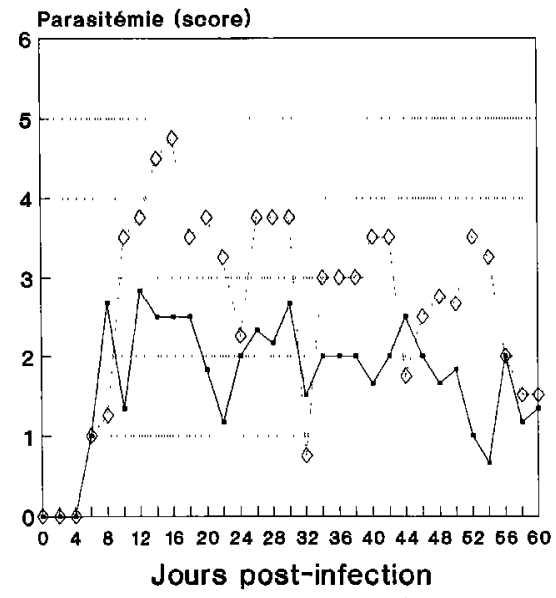

(3)

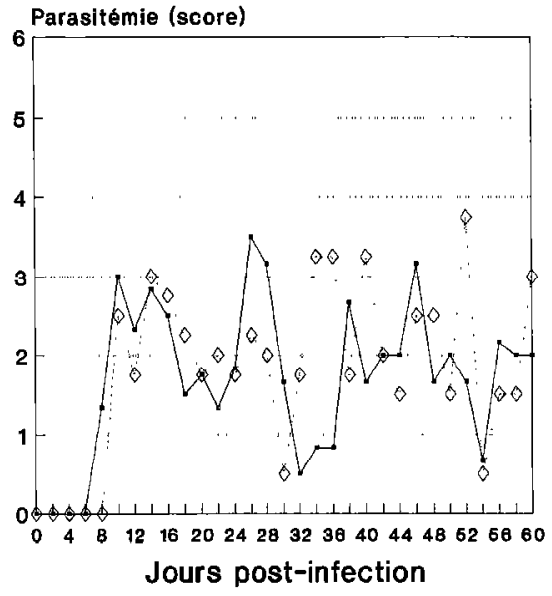

(2)

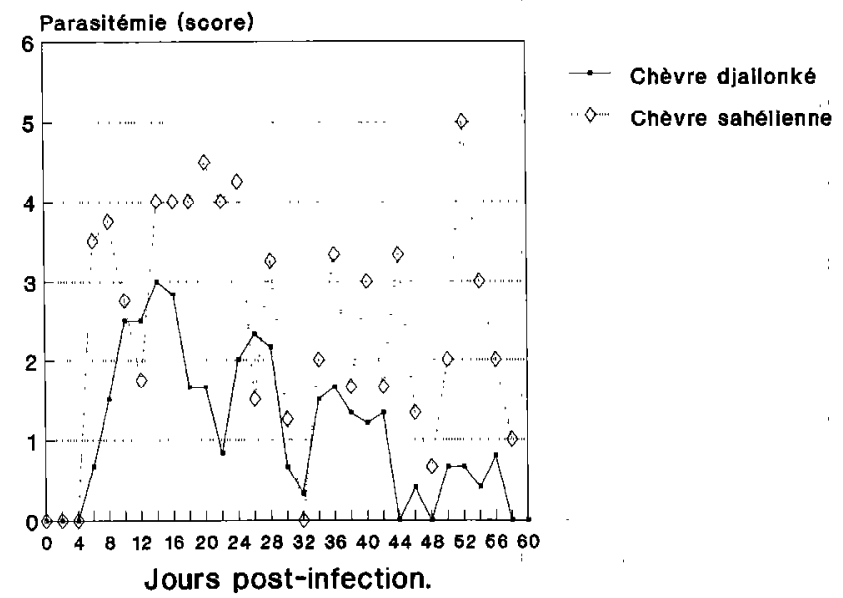

(4)

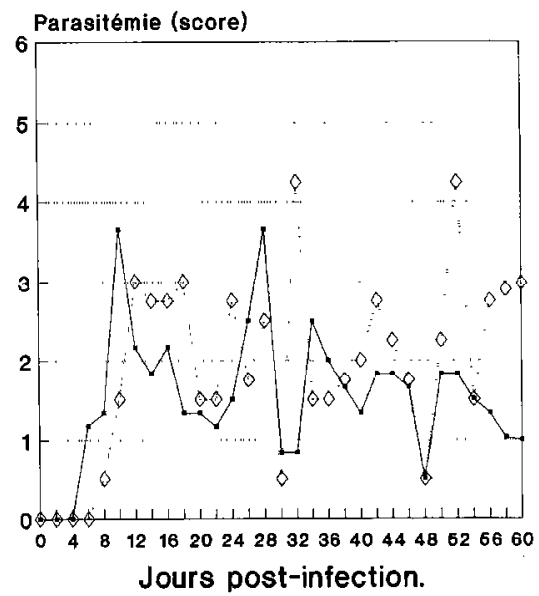

- Ghèvre djallonké

- Chèvre sahélienne

Figure 3 : Évolutions de la parasitémie. (1) et (2) : infection à $\mathrm{T}$. vivax; (3) et (4) : infection à $\mathrm{T}$. congolense.

TABLEAU III Nombre moyen de jours de parasitémie détectable.

\begin{tabular}{|c|c|c|c|}
\hline Lots & Infection & $\begin{array}{l}\text { Nombre moyen de jours } \\
\text { de parasitémie détectable (p. 100) }\end{array}$ & $\begin{array}{c}\text { Signification } \\
\text { statistique }\end{array}$ \\
\hline $\begin{array}{l}\text { Mouton Djallonké } \\
\text { Mouton Peul du Sahel }\end{array}$ & $\begin{array}{l}T . \text { vivax } \\
T . \text { vivax }\end{array}$ & $\begin{array}{l}48,79 \pm 9,1 \\
79,61 \pm 4\end{array}$ & $S(p<0,01)$ \\
\hline $\begin{array}{l}\text { Mouton Djallonké } \\
\text { Mouton Peul du Sahel }\end{array}$ & $\begin{array}{l}\text { T. congolense } \\
T . \text { congolense }\end{array}$ & $\begin{array}{l}52,81 \pm 15 \\
67,68 \pm 7,3\end{array}$ & $S(p<0,05)$ \\
\hline $\begin{array}{l}\text { Chèvre Djallonké } \\
\text { Chèvre Peul du Sahel }\end{array}$ & $\begin{array}{l}T \text {. vivax } \\
T \text {. vivax }\end{array}$ & $\begin{array}{r}36,5 \pm 2,1 \\
67,78 \perp 7,6\end{array}$ & $S(p<0,01)$ \\
\hline $\begin{array}{l}\text { Chèvre Djallonké } \\
\text { Chèvre Peul du Sahel }\end{array}$ & $\begin{array}{l}T \text {. congolense } \\
T \text {. congolense }\end{array}$ & $\begin{aligned} 53,58 & \pm 6 \\
57,3 & \pm 4,5\end{aligned}$ & NS \\
\hline
\end{tabular}


(1)

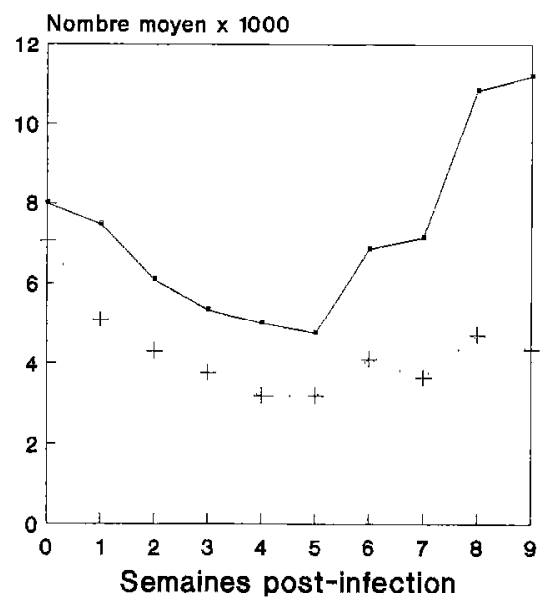

(3)

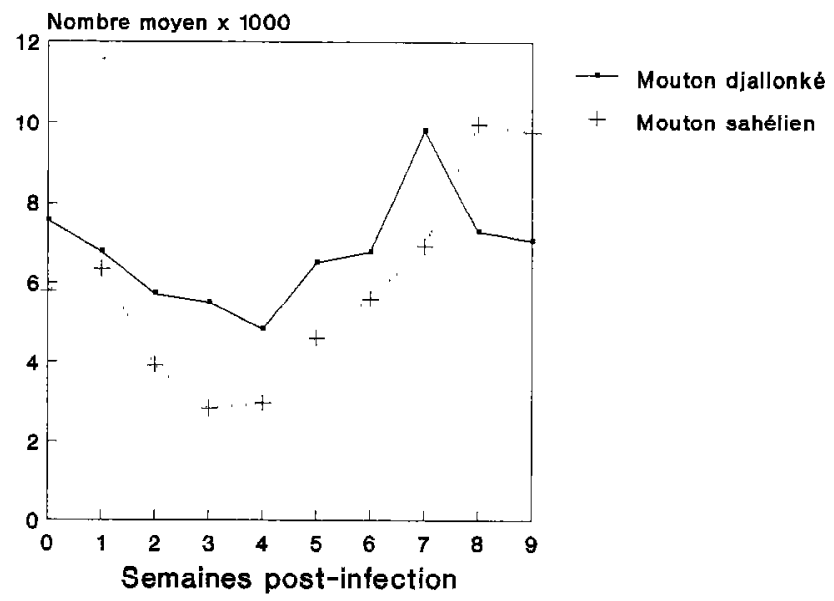

(2)

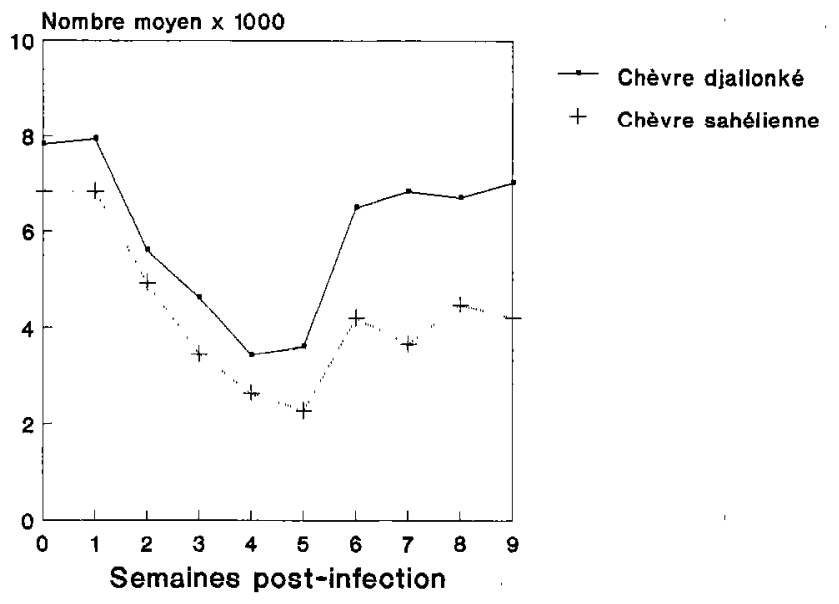

(4)

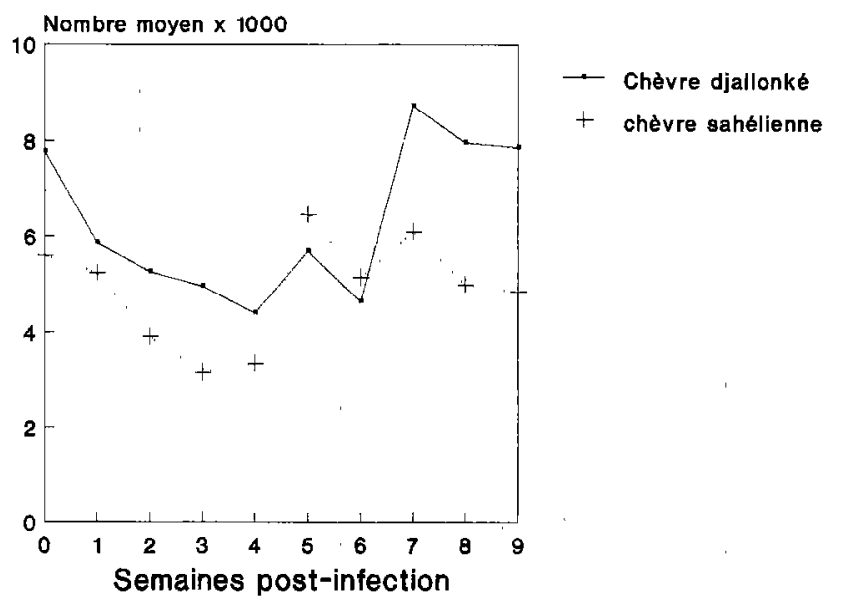

Figure 4 : Évolutions des concentrations leucocytaires. (1) et (2) : infection à $\mathbf{T}$. vivax ; (3) et (4) : infection à T. congolense.

\section{Cinétique des anticorps}

Les taux d'anticorps IgM et IgG augmentent durant les trois premières semaines post-infection puis semblent se stabiliser dans tous les lots. Pour l'infection par $T$. vivax, les animaux Peul ont des titres supérieurs à ceux de race Djallonké. L'inverse a été observé pour les infections à $T$. congolense.

\section{DISCUSSION}

Les comparaisons d'ordres clinique, hématologique et parasitologique entre les races étudiées dans nos conditions expérimentales, montrent uniquement pour $T$. vivax une différence significative entre moutons et chc̀vres do race Djallonké d'une part et de race Peul du Sahel d'autre part. En effet, les manifestations cliniques sont plus sévères chez les Peul. Cela se traduit notamment sur le plan de la perte de poids qui est deux fois plus importante, et des mortalités enregistrées. II en est de même de l'hématocrite et de la concentration leucocytaire dont les altérations sont plus prononcées chez les Peul que chez les Djallonké. Ce meilleur comportement des Djallonké semble illustrer leur plus grande aptitude à contrôler la parasitémie et à résister aux effets de l'anémie. Des facteurs innés et/ou acquis pourraient être à l'origine de cette aptitude. Mais il est impossible de préciser leur importance relative, du fait de l'exposition préalable des Djallonké à la trypanosomose. En effet, il a été montré chez les bovins trypanotolérants (7), qu'une résistance acquise grâce à une exposition préalable pouvait renforcer la résistance innée naturelle de ces animaux. Cette étude ne saurait illustrer le caractère inné ou acquis de la trypanotolérance chez les petits ruminants. II s'agit de confirmer expérimentalement des observations de terrain. 
Les mêmes comparaisons d'ordres clinique, hématologique et parasitologique appliquées aux lots infectés à T.congolense ne montrent pas de différence significative entre Djallonké et sahéliens. Ce résultat pourrait provenir de la faible virulence observée du stock de $T$. congolense. Des comparaisons similaires devraient être réalisées avec d'autres stocks.

Cette étude est à rapprocher de celle de BUENGENER et MEHLITZ (2), qui ont constaté expérimentalement une bonne résistance des chèvres naines du Cameroun à l'égard de $T$. vivax et de $T$. congolense. Ces mêmes chèvres étaient tuées par un stock de $T$. brucei. Elle se rapproche également de l'étude de WHITELAW et al. (9) au Kenya, qui montrent que les chèvres locales de race Galla, les Nubian et Toggenburg sont toutes sensibles à l'infection expérimentale à $T$. congolense.

Des épreuves d'infections expérimentales réalisées par TOURÉ et al. (8) au Sénégal montrent par contre une bonne résistance des moutons Djallonké par rapport aux moutons Peul pour $T$. congolense et non pour $T$. vivax. Leur expérimentation a duré 23 semaines contre 16 dans cette étude dont 8 seulement pour les paramètres classiquement comparés pour l'étude de trypanotolérance. Ce travail de comparaison mériterait donc d'être repris sur des périodes plus longues avec des stocks de trypanosomes de virulences variées.

\section{CONCLUSION}

Ces études en conditions contrôlées montrent, seulement pour l'infection par $T$. vivax, une bonne résistance des moutons et chèvres Djallonké par rapport à ceux du Sahel. En revanche, les races Djallonké et du Sahel ne semblent pas avoir de comportement significativement différent pour l'infection par T. congolense. Le meilleur comportement des Djallonké pourrait être lié à des mécanismes physiologiques innés et/ou aquis. Une étude similaire avec des animaux nés et élevés dans une étable sous moustiquaire mériterait d'être conduite pour préciser leur importance relative.

\section{BIBLIOGRAPHIE}

1. BOCQUENTIN (R.), DUVALLET (G.). Amélioration de la reproductibilité du test ELISA adapté à la détection d'anticorps anti-Trypanosoma congolense chez les bovins. Revue Élev. Méd. vét. Pays trop.,1990, 43 (2) : $179-186$.

2. BUENGENER (W.), MEHLITZ (D.). Experimental Trypanosoma infections in Cameroon dwarf goats: histopathological observations.Tropenmed Parasit.,1976, 27 (4) : 405-410.

3. Centre de recherches sur les trypanosomoses animales (CRTA). Rapport succinct d'activités, 1989. Bobo-Dioulasso, CRTA. P. 25-26.

4. COLES (E.H.). Le laboratoire en clinique vétérinaire. Paris, Vigot Frère, 1979.

5. DISSET (R.). L'élevage au Burkina Faso. III. Étude particulière de l'élevage des petits ruminants au Burkina Faso. Rapport de mission de consultation effectuée du 21 janvier au 15 février 1985 pour le compte de l'unité sous-régionale d'appui au développenent dans le cadre du programme FAO de lutte contre la trypanosomiase animale et de mise en valeur des zones concernées (GCP/RAF/191/ITA). Ouagadougou, FAO, 1985. P. 3435 .

6. MURRAY (M.), MURRAY (P.K.), McINTYRE (W.I.M). An improved parasitological technique for diagnosis of African trypanosomiasis. Trans. R. Soc. trop. Med. Hyg.,1977, 71 : 325-326.

7. PALING (R.W.), MOLOO (S.K.), SCOTT (J.R.), GETTINBY (G.), McODIMA (F.A.), MURRAY (M.). Susceptibility of N'Dama and Boran cattle to sequential challenges with tsetse-transmitted clones of Trypanosoma congolense. Parasit. Immun., 1991, 13 (4) : 427-445.

8. TOURÉ (S.M.), SEYE (M.), MBENGUE (M.), DIEYE (T.). Trypanotolérance : Étude de pathologie comparée entre moutons Djallonké et moutons Peulh du Sahel. In : Actes de la17e Réunion du conseil scientifique international de la Recherche sur les trypanosomoses et leur contrôle, Arusha, 1981. Nairobi, Eleza Services Ltd, 1983. (OAU/STRC, Publication $n^{\circ}$ 112)

9. WHI'IELAW (D.D.), KAAYA (G.P.), MOULTON (J.E.), MOLOO (S.K.), MURRAY (M). Susceptibility of different breeds of goats in Kenya to experimental infection with Trypanosoma congolense. Trop. Anim. Hith Prod., 1985, 17 (3) : 155-165. 


\section{Z. Bengaly P.H. Clausen H. Boly A. Kawne G. Duvallet}

BENGALY (Z.), CLAUSEN (P.H.), BOLY (H.), KANWE (A.), DUVALLET (G.). Experimental infection of small ruminants with trypanosomes : comparison of Djallonké and Sahelian breeds in Burkina Faso. Revue Élev. Méd. vét. Pays trop.., 1993, 46 (4) : 563-570

Twenty-four dwarf Djallonké sheep and goats, and 16 Sahelian Fulani sheep and goats, were inoculated with strains of Trypanosoma vivax and Tryapanosoma congolense to compare their degree of susceptibility to trypanosomosis. One animal from each breed was used as a control. Anaemia was observed in all inoculated animals. In the group of animals inoculated with $T$. vivax, 1 Djallonké goat out of 6,3 Sahelian goats out of 4 and 2 Fulani sheep out of 4 died within the experimental period of 16 weeks. One single Sahelian Fulani sheep out of 4 died among animals inoculated with $T$. congolense. During a period of 8 weeks, no significant difference was observed between Djallonké and Fulani Sahelian in the decrease of body weight and leucocyte count. Results in this study show a better resistance of Djallonké sheep and goats to infection by $T$. vivax, whereas there was no significant difference between all sheep and goats inoculated with T.congolense.

Keys-words : Goat Sheep Djallonké goat - Sahelian Fulani goat-Djallonké sheep - Sahelian Fulani sheep - Trypanosomosis - Trypanosoma congolense - Trypanosomosa vivax - Trypanotolerance - Experimental infection - Burkina Faso.
BENGALY (Z.), CLAUSEN (P.H.), BOLY (H.), KANWE (A.), DUVALLET (G.). Comparación de la tripanosomosis experimental en distintas razas de pequeños rumiantes de Burkina Faso. Revue Élev. Méd. vét. Pays trop.., 1993, 46 (4) : 563-570

Para comparar los diferentes grados de sensibilidad a las tripanosomosis, 24 ovinos y caprinos de raza Djallonké enana y 16 ovinos y caprinos Peul del Sahel se inocularon con cepas de Trypanosoma vivax y de Trypanosoma congolense. Un animal de cada raza se utilizó como testigo. Todos los animales inoculados presentaron anemia. Durante las 16 semanas de experimentación, la mortalidad por Trypanosoma vivax fué de una cabra Djallonké sobre seis, tres cabras sobre cuatro y dos ovinos sobre cuatro de raza Peul del Sahel. Para Trypanosoma congolense se observó un sólo caso de mortalidad sobre cuatro en el hato de ovinos Peul del Sahel. Durante ocho semanas no se oloservaron diferencias significativas para la pérdida de peso y la concentración leucocitaria entre Djallonké y Peul del Sahel. Este estudio evidencia la buena resistencia de los ovinos y caprinos Djallonké a la infección por Trypanosoma vivax. Por el contrario, no se encontró una diferencia significativa entre las dos razas de ovinos y caprinos hacia el Trypanosoma congolense.

Palabras claves : Caprino - Ovino - Caprino Djallonké - Caprino Peul Ovino Djallonké - Ovino Peul - Tripanosomosis - Trypanosoma congolense - Trypanosoma vivax - Tripanotolerancia - Infección experimental Burkina Faso. 